\title{
Effect of Radiative Heat-Loss Function and Finite Larmor Radius Corrections on Jeans Instability of Viscous Thermally Conducting Self-Gravitating Astrophysical Plasma
}

\author{
Sachin Kaothekar ${ }^{1,2}$ and R. K. Chhajlani' \\ ${ }^{1}$ School of Studies in Physics, Vikram University, Madhya Pradesh, Ujjain 456010, India \\ ${ }^{2}$ Department of Physics, Mahakal Institute of Technology, Madhya Pradesh, Ujjain 456664, India
}

Correspondence should be addressed to Sachin Kaothekar, sackaothekar@gmail.com

Received 3 April 2012; Accepted 15 June 2012

Academic Editors: C. W. Engelbracht, A. Ferrari, F. Fraschetti, I. Goldman, and C. Meegan

Copyright ( $) 2012$ S. Kaothekar and R. K. Chhajlani. This is an open access article distributed under the Creative Commons Attribution License, which permits unrestricted use, distribution, and reproduction in any medium, provided the original work is properly cited.

\begin{abstract}
The effect of radiative heat-loss function and finite ion Larmor radius (FLR) corrections on the self-gravitational instability of infinite homogeneous viscous plasma has been investigated incorporating the effects of thermal conductivity and finite electrical resistivity for the formation of a star in astrophysical plasma. The general dispersion relation is derived using the normal mode analysis method with the help of relevant linearized perturbation equations of the problem. Furthermore the wave propagation along and perpendicular to the direction of external magnetic field has been discussed. Stability of the medium is discussed by applying Routh Hurwitz's criterion. We find that the presence of radiative heat-loss function and thermal conductivity modify the fundamental Jeans criterion of gravitational instability into radiative instability criterion. From the curves we see that temperature dependent heat-loss function, FLR corrections and viscosity have stabilizing effect, while density dependent heat-loss function has destabilizing effect on the growth rate of self-gravitational instability. Our result shows that the FLR corrections and radiative heat-loss functions affect the star formation.
\end{abstract}

\section{Introduction}

The problem of self-gravitational instability is widely investigated due to its relevance to the fragmentation of interstellar medium and its role in star formation. Also, the selfgravitational instability of molecular clouds is connected to the cloud collapse and star formation. Hayashi [1] has discussed the problem of evolution of protostars and discusses the different phase of formation of protostar in connection with variation of temperature and density. Shu et al. [2] have investigated the problem of star formation in molecular clouds and concluded that the star formation occurs mainly in four phases. In this problem, the self-gravitational instability of dust and gas plays important role. Draine and Mckee [3] have studied the theory of interstellar shocks. Mckee and Ostriker [4] have discussed the theory of star formation. They concluded that the key dynamical processes involved in star formation are turbulence, magnetic fields, and selfgravity. In this connection, the gravitational instability of infinite homogeneous self-gravitating magnetized and rotating plasma is also discussed by Chandrasekhar [5]. Several authors (Pacholczyk and Stodolkiewicz [6], Nayyar [7], and Shaikh et al. [8]) have investigated the problem of gravitational instability of plasma with different physical parameters such as viscosity, finite electrical conductivity, Hall current, thermal conductivity, magnetic field, and rotation. Yang et al. [9] have investigated the problem of largescale gravitational instability and star formation. Borah and Sen [10] have investigated the gravitational instability of partially ionized molecular clouds considering the effects of electrons, ions, and charged dust grains. Avinash et al. [11] have studied the dynamics of self-gravitating dust clouds and the formation of planetesimals. Thus we find that a large number of problems are discussed for self-gravitating dusty and nondusty plasma with different parameters under various assumptions due to its importance in star formation and in many astrophysical situations. 
Along with this, in above discussed problems, the effect of finite ion Larmor radius is not considered. In many astrophysical situations such as in interstellar and interplanetary plasmas the approximation of zero Larmor radius is not valid. Several authors (Roberts and Taylor [12], Jeffery and Taniuti [13], Jukes [14], and Vandakurov [15]) have pointed out the importance of finite ion Larmor radius (FLR) effects in the form of magnetic resistivity, on the plasma instability. Sharma [16] has shown the stabilizing effect of FLR on gravitational instability of rotating plasma. Bhatia and Chhonkar [17] have investigated the stabilizing effect of FLR on the instability of a rotating and self-gravitating plasma. Herrnegger [18] has studied the effects of collision and gyroviscosity on gravitational instability in a two-component plasma and concluded that the critical wave number becomes smaller with increasing gyroviscosity for finite Alfven numbers and showed that Jeans criterion is changed by FLR for wave propagating perpendicular to magnetic field. Vaghela and Chhajlani [19] have investigated the stabilizing effect of FLR on magneto-gravitational stability of resistive plasma through porous medium with thermal conduction, but they neglect the effect of radiative heat-loss function on gravitational instability. Chhajlani and Parihar [20] have carried out the stabilizing effect of FLR on magnetogravitational instability of anisotropic plasma with generalized polytrope laws. Ferraro [21] has shown the stabilizing effect of FLR on magneto-rotational instability. Recently Sandberg et al. [22] have investigated the stabilizing effect of FLR on the coupled trapped electron and ion temperature-gradient modes. More recently Devlen and Pekünlü [23] have studied the effect of FLR on weakly magnetized, dilute plasma. Thus FLR effect is an important factor in discussion of self-gravitational instability and other hydrodynamic instability.

In addition to this it is well known that thermal and radiative effects do play an important role in the stability investigations. The thermal instability arising due to various heat-loss mechanisms may be the cause of astrophysical condensation and the formation of large and small objects. Several authors (Field [24], Hunter [25], Ibanez [26], Kim and Narayan [27], Radwan [28], Menou et al. [29], and Inutsuka et al. [30]) have investigated the phenomenon of thermal instability arising due to heat-loss mechanism in plasma. Shadmehri and Dib [31] have discussed the thermal instability in magnetized partially ionized plasma with charged dust particles and radiative cooling function. Shaikh et al. [32] have investigated the Jeans gravitational instability of thermally conducting plasma in a variable magnetic field with Hall current, finite conductivity, and viscosity, but they neglect the effect of FLR corrections and radiative heat-loss function on gravitational instability. Aggarwal and Talwar [33] have discussed magnetothermal instability in a rotating gravitating fluid, but they neglect the effect of FLR correction on radiative instability. Bora and Talwar [34] have investigated the magnetothermal instability with finite electrical resistivity and Hall current, but they neglect the effect of FLR corrections and viscosity on radiative instability. Recently ElSayed and Mohamed [35] have discussed the gravitational instability of rotating viscoelastic partially ionized plasma in the presence of oblique magnetic field and Hall current.
More recently Kaothekar and Chhajlani [36] have carried out the problem of gravitational instability of radiative plasma with FLR corrections.

In the light of above work, we find that all these authors have studied the problem of gravitational instability with different combinations of these parameters, but none studied the joint effect of all the parameters together with radiative effects. We also find that none of the above authors has tried to explore Jeans condition for viscous and nonviscous plasma with FLR corrections, radiative heat-loss function, and thermal conductivity. The problem given by Kaothekar and Chhajlani [36] is briefly discussed with permeability parameter, but in present paper we have made a detailed analysis of the problem of self-gravitational instability with all the considered physical parameters, without including the effect of permeability to give a better insight in real problem. Therefore in the present work self-gravitational instability of magnetized plasma with FLR corrections, radiative heat-loss function, viscosity, thermal conductivity, and finite electrical resistivity for self-gravitating configuration is studied. We also wish to explore the importance of viscous and nonviscous system and its impact on the self-gravitational instability of plasma in connection with FLR correction, radiative heat-loss function, thermal conductivity, and finite electrical resistivity. The stability of the system is discussed by applying Routh-Hurwitz criterion. The above work is applicable to formation of stars in astrophysical plasma.

\section{Basic Equations of the Problem and Perturbation}

Let us consider an infinite homogeneous, self-gravitating, radiating, thermally conducting, and viscous plasma of finite electrical resistivity, including the effect of finite ion Larmor radius (FLR) corrections in the presence of magnetic field $\mathbf{H}(0,0, H)$. The equations of the problem with these effects are written as

$$
\begin{gathered}
\frac{d \mathbf{u}}{d t}=-\frac{1}{\rho} \nabla p+\nabla U-\frac{\nabla \cdot \mathbf{P}}{\rho}+\frac{1}{4 \pi \rho}(\nabla \times \mathbf{H}) \times \mathbf{H}+v \nabla^{2} \mathbf{u}, \\
\frac{1}{\gamma-1} \frac{d p}{d t}-\frac{\gamma}{\gamma-1} \frac{p}{\rho} \frac{d \rho}{d t}+\rho L-\nabla \cdot(\lambda \nabla T)=0, \\
p=\rho R T, \\
\frac{d \rho}{d t}+\rho \nabla \cdot \mathbf{u}=0, \\
\nabla^{2} U=-4 \pi G \rho, \\
\frac{\partial \mathbf{H}}{\partial t}=\nabla \times(\mathbf{u} \times \mathbf{H})+\eta \nabla^{2} \mathbf{H}, \\
\nabla \cdot \mathbf{H}=0,
\end{gathered}
$$

where $p, \rho, v, T, \eta, \lambda, U, G, R$, and $\gamma$ denote the fluid pressure, density, kinematic viscosity, temperature, electrical resistivity, thermal conductivity, gravitational potential, gravitational constant, gas constant, and ratio of two specific 
heats respectively. $L(\rho, T)$, the radiative heat-loss function, depends on local values of density and temperature of the fluid. The operator $(d / d t)$ is the substantial derivative given as $(d / d t)=(\partial / \partial t+\mathbf{u} \cdot \nabla)$.

The components of pressure tensor $\mathbf{P}$, considering the finite ion gyration radius for the magnetic field along $z$-axis as given by Roberts and Taylor [12], are

$$
\begin{gathered}
P_{x x}=-\rho v_{0}\left(\frac{\partial u_{y}}{\partial x}+\frac{\partial u_{x}}{\partial y}\right), \quad P_{y y}=\rho v_{0}\left(\frac{\partial u_{y}}{\partial x}+\frac{\partial u_{x}}{\partial y}\right), \\
P_{z z}=0, \quad P_{x y}=P_{y x}=\rho v_{0}\left(\frac{\partial u_{x}}{\partial x}-\frac{\partial u_{y}}{\partial y}\right), \\
P_{x z}=P_{z x}=-2 \rho v_{0}\left(\frac{\partial u_{y}}{\partial z}+\frac{\partial u_{z}}{\partial y}\right), \\
P_{y z}=P_{z y}=2 \rho v_{0}\left(\frac{\partial u_{z}}{\partial x}+\frac{\partial u_{x}}{\partial z}\right) .
\end{gathered}
$$

The parameter $v_{0}$ has the dimensions of the kinematic viscosity and defined as $v_{0}=\Omega_{L} R_{L}^{2} / 4$, where $R_{L}$ is the ionLarmor radius, and $\Omega_{L}$ is the ion gyration frequency. The perturbation in fluid pressure, density, temperature, velocity, magnetic field, heat-loss function, and gravitational potential is given as $\delta p, \delta \rho, \delta T, \mathbf{u}\left(u_{x}, u_{y}, u_{z}\right), \mathbf{h}\left(h_{x}, h_{y}, h_{z}\right), L$, and $\delta U$, respectively. The perturbation state is given as

$$
\begin{gathered}
p=p_{0}+\delta p, \quad \rho=\rho_{0}+\delta \rho, \\
T=T_{0}+\delta T, \quad \mathbf{u}=\mathbf{u}_{0}+\mathbf{u} \quad\left(\text { with } \mathbf{u}_{0}=0\right), \\
\mathbf{H}=\mathbf{H}_{0}+\mathbf{h}, \quad L=L_{0}+L \quad\left(\text { with } L_{0}=0\right), \\
U=U_{0}+\delta U .
\end{gathered}
$$

Suffix "0" represents the initial equilibrium state, which is independent of space and time.

\section{Dispersion Relation}

We linearize the basic set of MHD equations by substituting (3) in (1)-(2), and we assume the variation of perturbed quantities as

$$
\exp \left(i k_{x} x+i k_{z} z+i \sigma t\right)
$$

where $\sigma$ is the frequency, and $k_{x}$ and $k_{z}$ are the wave numbers of the perturbations along $x$ and $z$ axes. Using (4) in linearized perturbed form of (1) with (2), we get four equations governing the perturbations of velocity and the condensation of medium. These four equations can be written in the following determinant form:

$$
\begin{gathered}
{\left[\begin{array}{cccc}
P & F & 0 & \frac{i k_{x}}{k^{2}} \Omega_{T}^{2} \\
-F & Q & -2 v_{0} k_{x} k_{z} & 0 \\
0 & 2 v_{0} k_{x} k_{z} & M & \frac{i k_{z}}{k^{2}} \Omega_{T}^{2} \\
i k_{x} \frac{V^{2} k^{2}}{d} & i k_{x} v_{0}\left(k_{x}^{2}+4 k_{z}^{2}\right) & 0 & -R
\end{array}\right]} \\
\times\left[\begin{array}{c}
u_{x} \\
u_{y} \\
u_{z} \\
s
\end{array}\right]=0 .
\end{gathered}
$$

We have made following substitutions:

$$
\begin{gathered}
i \sigma=\omega, \quad R=\omega^{2}+\omega v k^{2}+\Omega_{T}^{2}, \\
F=v_{0}\left(k_{x}^{2}+2 k_{z}^{2}\right), \quad d=\left(\omega+\eta k^{2}\right), \\
M=\omega+v k^{2}, \quad P=M+\frac{V^{2} k^{2}}{d}, \\
Q=M+\frac{V^{2} k_{z}^{2}}{d}, \quad L_{\rho}=\left(\frac{\partial L}{\partial \rho}\right)_{T}, \\
V^{2}=\frac{H^{2}}{4 \pi \rho}, \quad \Omega_{T}^{2}=\frac{\Omega_{I}^{2}+\omega \Omega_{j}^{2}}{\omega+B}, \\
\Omega_{j}^{2}=c^{2} k^{2}-4 \pi G \rho, \quad \Omega_{I}^{2}=k^{2} A-4 \pi G \rho B, \\
\left.\frac{\partial L}{\partial T}\right)_{\rho} \quad A=(\gamma-1)\left(T L_{T}-\rho L_{\rho}+\frac{\lambda k^{2} T}{\rho}\right), \\
B=(\gamma-1)\left(\frac{T \rho L_{T}}{p}+\frac{\lambda k^{2} T}{p}\right),
\end{gathered}
$$

where $c=(\gamma p / \rho)^{1 / 2}$ is the adiabatic velocity of sound in the medium, $c^{\prime}=(p / \rho)^{1 / 2}$ is the isothermal velocity of sound in the medium, and $s=\delta \rho / \rho$ is the condensation of the medium.

The general dispersion relation can be obtained from the determinant of matrix of (5) is

$$
\begin{aligned}
& {\left[\omega+v k^{2}+\frac{V^{2} k^{2}}{\omega+\eta k^{2}}\right]\left(\omega^{2}+\omega v k^{2}+\frac{\Omega_{I}^{2}+\omega \Omega_{j}^{2}}{\omega+B}\right)} \\
& \quad \times\left\{4 v_{0}^{2} k_{x}^{2} k_{z}^{2}+\left(\omega+v k^{2}\right) \times\left[\omega+v k^{2} \frac{V^{2} k_{z}^{2}}{\omega+\eta k^{2}}\right]\right\} \\
& \quad-\left(k_{x}^{2}+4 k_{z}^{2}\right)\left[\omega+v k^{2}+\frac{V^{2} k^{2}}{\omega+\eta k^{2}}\right] \\
& \quad \times\left(\frac{2 v_{0}^{2} k_{x}^{2} k_{z}^{2}}{k^{2}}\right)\left(\frac{\Omega_{I}^{2}+\omega \Omega_{j}^{2}}{\omega+B}\right) \\
& \quad+v_{0}^{2}\left(k_{x}^{2}+2 k_{z}^{2}\right)^{2}\left[\omega^{2}+\omega v k^{2}+\frac{\Omega_{I}^{2}+\omega \Omega_{j}^{2}}{\omega+B}\right]\left(\omega+v k^{2}\right)
\end{aligned}
$$




$$
\begin{aligned}
& +\left(\frac{\Omega_{I}^{2}+\omega \Omega_{j}^{2}}{\omega+B}\right)\left(\frac{V^{2}}{\omega+\eta k^{2}}\right) \times 2 v_{0}^{2} k_{x}^{2} k_{z}^{2}\left(k_{x}^{2}+2 k_{z}^{2}\right) \\
& -\left(\omega+v k^{2}\right) \frac{v_{0}^{2} k_{x}^{2}}{k^{2}}\left(\frac{\Omega_{I}^{2}+\omega \Omega_{j}^{2}}{\omega+B}\right)\left(k_{x}^{2}+2 k_{z}^{2}\right)\left(k_{x}^{2}+4 k_{z}^{2}\right) \\
& -\left(\omega+v k^{2}\right) \\
& \times\left[\omega+v k^{2}+\frac{V^{2} k_{z}^{2}}{\omega+\eta k^{2}}\right]\left(\frac{V^{2} k_{x}^{2}}{\omega+\eta k^{2}}\right)\left(\frac{\Omega_{I}^{2}+\omega \Omega_{j}^{2}}{\omega+B}\right) \\
& -4 v_{0}^{2} k_{x}^{4} k_{z}^{2}\left(\frac{V^{2}}{\omega+\eta k^{2}}\right)\left(\frac{\Omega_{I}^{2}+\omega \Omega_{j}^{2}}{\omega+B}\right)=0 .
\end{aligned}
$$

The dispersion relation (7) represents the simultaneous inclusion of FLR corrections, radiative heat-loss function, thermal conductivity, viscosity, magnetic field, and finite electrical conductivity on self-gravitational instability of plasma. In absence of radiative heat-loss function, the general dispersion relation (7) is identical to that of Vaghela and Chhajlani [19] for $\left(\varepsilon=1\right.$ and $\left.K_{1}=\infty\right)$ in their case. In absence of radiative heat-loss function, thermal conductivity, finite electrical resistivity, and viscosity the general dispersion relation (7) is identical to Sharma [16] for nonrotational case. In absence of FLR corrections and radiative heatloss function the general dispersion relation (7) is identical to Shaikh et al. [32] neglecting Hall current in their case. In absence of FLR corrections and viscosity dispersion relation (7) is identical to Bora and Talwar [34] neglecting Hall current and electron inertia in their case. Also in absence of FLR corrections, viscosity and finite conductivity dispersion relation (7) reduces to that obtained by Field [24] for nongravitating medium. The dispersion relation (7) is different from (9) of Kaothekar and Chhajlani [36].

Thus we have obtained the modified dispersion relation of self-gravitational instability including the combined effect of FLR corrections, radiative heat-loss function, viscosity, thermal conductivity, finite electrical resistivity, and magnetic field. Now we discuss the general dispersion relation (7) for longitudinal and transverse wave propagation.

\section{Discussion}

4.1. Longitudinal Propagation $\left(k_{x}=0, k_{z}=k\right)$. For this case, we assume all the perturbations are longitudinal to the direction of magnetic field (i.e., $k_{z}=k, k_{x}=0$ ). The dispersion relation $(7)$ reduces to

$$
\begin{aligned}
\left(\omega+v k^{2}\right) \times & \left\{4 v_{0}^{2} k^{4}+\left[\omega+v k^{2}+\frac{V^{2} k^{2}}{\omega+\eta k^{2}}\right]^{2}\right\} \\
\times & {\left[\omega^{2}+\omega v k^{2}+\frac{\omega\left(c^{2} k^{2}-4 \pi G \rho\right)}{\omega+(\gamma-1)\left(T \rho L_{T} / p+\lambda k^{2} T / p\right)}\right.} \\
& \left.+\frac{(\gamma-1)\left\{k^{2}\left(T L_{T}-\rho L_{\rho}+\lambda k^{2} T / p\right)-4 \pi G \rho\left(T \rho L_{T} / p+\lambda k^{2} T / p\right)\right\}}{\omega+(\gamma-1)\left(T \rho L_{T} / p+\lambda k^{2} T / p\right)}\right]=0 .
\end{aligned}
$$

The above dispersion relation shows the combined effect of FLR corrections, radiative heat-loss function, thermal conductivity, viscosity, magnetic field strength, and finite electrical resistivity on self-gravitation instability of plasma. On multiplying all the components of (8), we get the dispersion relation, which is an equation of degree eight in $\omega$, and it is cumbersome to write such a lengthy equation. If we remove the effect of FLR corrections and viscosity in the above relation, then we recover the relation given by Bora and Talwar [34] excluding Hall current and electron inertia in their case. Hence the above dispersion relation is the modified form of equation (21) of Bora and Talwar [34] due to the inclusion of FLR corrections and viscosity in our case and by neglecting Hall current and electron inertia in their case for longitudinal propagation in dimensional form. But the condition of instability is unaffected by the presence of FLR correction and viscosity. Thus we conclude that FLR corrections and viscosity have no effect on the condition of instability, but presence of these parameters modifies the growth rate of instability in the present case. Hence these are the new findings in our case than that of Bora and Talwar
[34]. Also on comparing our dispersion relation (8) with dispersion relation (20) of Vaghela and Chhajlani [19] we find that two factors are the same, but the third factor is different and gets modified because of radiative terms.

The dispersion relation (8) has three different components, and our aim is to take out the physics involved in each component. So we discuss each component separately. The first component of the dispersion relation (8) gives

$$
\omega+v k^{2}=0
$$

This represents a damped mode modified by the presence of viscosity of the medium. Thus viscous force is capable of stabilizing the growth rate of the considered system. The above mode is unaffected by the presence of FLR correction, magnetic field strength, finite electrical resistivity, thermal conductivity, radiative heat-loss function, and selfgravitation. 
The second factor of (8) on simplification gives

$$
\begin{aligned}
\omega^{4} & +2\left(v k^{2}+\eta k^{2}\right) \omega^{3} \\
& +\left[\left(v k^{2}+\eta k^{2}\right)^{2}+2\left(V^{2} k^{2}+\eta k^{2} v k^{2}\right)+4 v_{0}^{2} k^{4}\right] \omega^{2} \\
& +\left[2\left(v k^{2}+\eta k^{2}\right) \times\left(V^{2} k^{2}+\eta k^{2} v k^{2}\right)+8 \eta k^{2} v_{0}^{2} k^{4}\right] \omega \\
& +\left(V^{2} k^{2}+\eta k^{2} v k^{2}\right)^{2}+4 \eta^{2} k^{4} v_{0}^{2} k^{4}=0 .
\end{aligned}
$$

The above dispersion relation represents the Alfven mode modified by the presence of FLR corrections, viscosity, and finite electrical resistivity. But it is independent of thermal conductivity, radiative heat-loss function, and selfgravitation. Equation (10) is a four-degree equation in power of $\omega$ having its all coefficients positive which is a necessary condition for the stability of the system. To achieve the sufficient condition the principal diagonal minors of Hurwitz matrix must be positive. On calculating we get all the principal diagonal minors positive. Hence (10) always represents stability.

The third component of the dispersion relation (8) on simplifying gives

$$
\begin{aligned}
\omega^{3}+ & {\left[v k^{2}+(\gamma-1)\left(\frac{T \rho L_{T}}{p}+\frac{\lambda k^{2} T}{p}\right)\right] \omega^{2} } \\
+ & {\left[(\gamma-1)\left(\frac{T \rho L_{T}}{p}+\frac{\lambda k^{2} T}{p}\right) v k^{2}+\left(c^{2} k^{2}-4 \pi G \rho\right)\right] \omega } \\
+ & \left\{k^{2}(\gamma-1)\left(T L_{T}-\rho L_{\rho}+\frac{\lambda k^{2} T}{\rho}\right)\right. \\
& \left.\quad-4 \pi G \rho(\gamma-1)\left(\frac{T \rho L_{T}}{p}+\frac{\lambda k^{2} T}{p}\right)\right\}=0
\end{aligned}
$$

The above equation represents the combined influence of radiative heat-loss function, thermal conductivity, and viscosity on the self-gravitational instability of plasma. But there is no effect of FLR corrections, finite electrical conductivity, and magnetic field on the self-gravitational instability of the considered system. If the constant term of cubic equation (11) is less than zero, this allows at least one positive real root which corresponds to the instability of the system. The condition of instability obtained from constant term of (11) is given as

$$
k^{2}\left(T L_{T}-\rho L_{\rho}+\frac{\lambda k^{2} T}{\rho}\right)<4 \pi G \rho\left(\frac{T \rho L_{T}}{p}+\frac{\lambda k^{2} T}{p}\right)
$$

This condition of instability is modified form of Jeans condition by inclusion of thermal conductivity and radiative heat-loss functions. The above condition of instability is independent of FLR corrections, finite electrical conductivity, magnetic field strength, and viscosity. The above inequality (12) is same as obtained by Bora and Talwar [34], and by Kaothekar and Chhajlani [36]. But Kaothekar and Chhajlani [36] have also considered the effect of permeability in their analysis. Here in the present problem we neglect the effect of permeability to give better insight in real problem. Thus in this mode the dispersion relation and the growth rate is modified due to FLR corrections and viscosity, but the condition of radiative instability is unaffected by the presence of FLR corrections and viscosity. The condition of instability (12) can be solved to yield the following modified critical Jeans wavelength:

$$
\lambda_{J 1}=\left[\frac{8 \pi}{\left\{\left(4 G \rho / c^{\prime 2}+\rho^{2} L_{\rho} / \pi \lambda T-\rho L_{T} / \pi \lambda\right) \pm\left[\left(4 G \rho / c^{\prime 2}+\rho^{2} L_{\rho} / \pi \lambda T-\rho L_{T} / \pi \lambda\right)^{2}+\left(16 G \rho^{2} / \pi \lambda c^{\prime 2}\right) L_{T}\right]^{1 / 2}\right\}}\right]^{1 / 2} .
$$

The medium is unstable for wave length $\lambda>\lambda_{J 1}$. Here it may be noted that the modified critical wave length involves the derivatives of temperature-dependent, densitydependent heat-loss functions and thermal conductivity of the medium.

In dispersion relation (8) first and second factors show wave propagation, but third factor shows instability. Thus to discuss the effect of each parameter on the growth rate of instability, we solve (11) numerically by introducing the following dimensionless quantities:

$$
\begin{gathered}
k^{*}=\frac{k c}{(4 \pi G \rho)^{1 / 2}}, \quad \omega^{*}=\frac{\omega}{(4 \pi G \rho)^{1 / 2}}, \\
v^{*}=\frac{v(4 \pi G \rho)^{1 / 2}}{c^{2}},
\end{gathered}
$$

$$
\begin{gathered}
L_{T}^{*}=\frac{(\gamma-1) \rho T L_{T}}{p(4 \pi G \rho)^{1 / 2}}, \quad L_{\rho}^{*}=\frac{(\gamma-1) \rho L_{\rho}}{c^{2}(4 \pi G \rho)^{1 / 2}}, \\
\lambda^{*}=\frac{(\gamma-1) T \lambda(4 \pi G \rho)^{1 / 2}}{p c^{2}} .
\end{gathered}
$$

The nondimensional form of (11) in terms of self-gravitation is given as

$$
\begin{aligned}
\omega^{* 3} & +\left(v^{*} k^{* 2}+L_{T}^{*}+\lambda^{*} k^{* 2}\right) \omega^{* 2} \\
& +\left[v^{*} k^{* 2}\left(L_{T}^{*}+\lambda^{*} k^{* 2}\right)+k^{* 2}-1\right] \omega^{*} \\
& +\left\{k^{* 2}\left[\frac{1}{\gamma}\left(L_{T}^{*}+\lambda^{*} k^{* 2}\right)-L_{\rho}^{*}\right]-\left(L_{T}^{*}+\lambda^{*} k^{* 2}\right)\right\}=0 .
\end{aligned}
$$




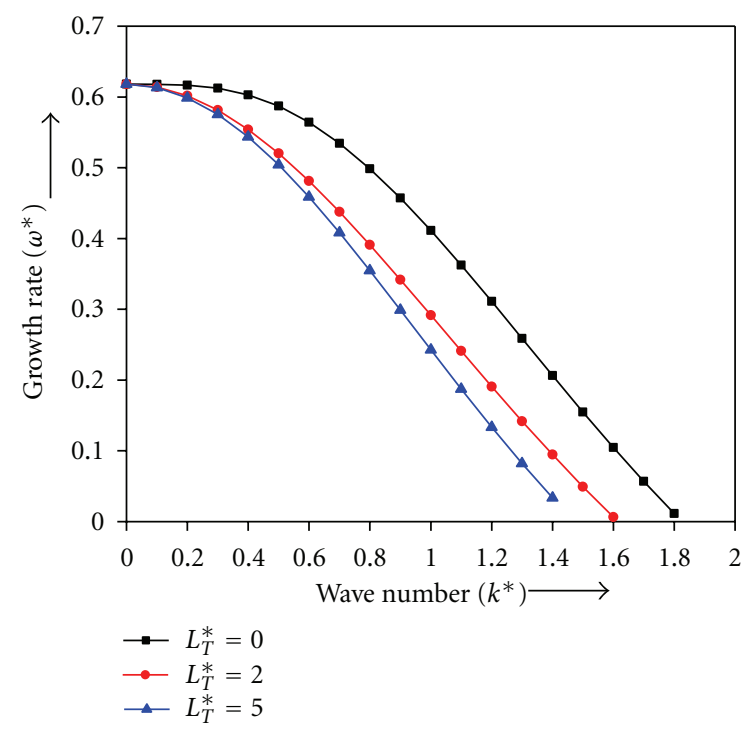

Figure 1: Growth rate (positive values of $\omega^{*}$ ) against wave number $k^{*}$ for three values of parameter $L_{T}^{*}=0.0,2.0,5.0$, keeping the other parameters fixed $L_{\rho}^{*}=1.0, \lambda^{*}=1.0, v^{*}=1.0$.

Here for calculations we take the numerical values corresponding to the conditions in interstellar molecular clouds for star formation as

$$
\begin{gathered}
\rho=1.7 \times 10^{-21} \mathrm{~kg} \mathrm{~m}^{-3} \\
G=6.658 \times 10^{-11}(\mathrm{~kg})^{-1} \mathrm{~m}^{3} \mathrm{~s}^{-2} \\
c^{2}=2.25 \times 10^{8} \mathrm{~m}^{2} \mathrm{~s}^{-2} \\
V^{2}=5.0 \times 10^{8} \mathrm{~m}^{2} \mathrm{~s}^{-2} .
\end{gathered}
$$

And the parameters are taken as nondimensional. Numerical calculations were performed to determine the roots of $\omega^{*}$ from dispersion relation (15), as a function of wave number $k^{*}$ for several values of the different parameters involved taking $\gamma=5 / 3$. Out of the three modes only one mode is unstable for which the calculations are presented in Figures $1-3$, where the growth rate $\omega^{*}$ (positive real value of $\omega$, after multiplying by $10^{16}$ ) has been plotted against the wave number $k^{*}$ (after multiplying by $10^{20}$ ) to show the dependence of the growth rate on the different physical parameters such as temperature-dependent heat-loss function $L_{T}^{*}$ (after multiplying by $10^{8}$ ), density-dependent heatloss function $L_{\rho}^{*}$ (after multiplying by $10^{8}$ ), and viscosity $v^{*}$ (after multiplying by $10^{24}$ ).

It is clear from Figure 1 that growth rate decreases with increasing temperature-dependent heat-loss function. Thus the effect of temperature-dependent heat-loss function is stabilizing. From Figure 2 we conclude that growth rate increases with increasing density-dependent heat-loss function. Thus the effect of density-dependent heat-loss function

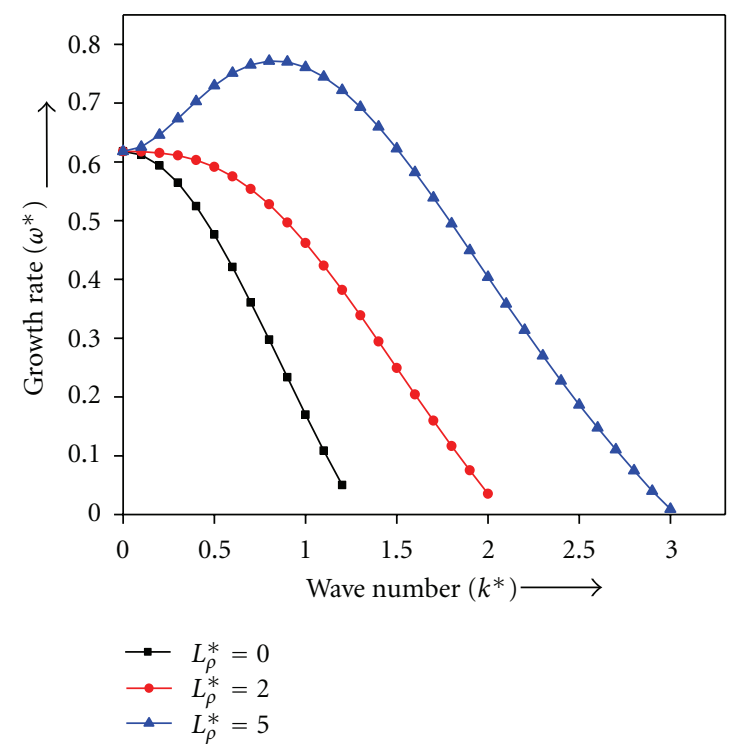

FIGURE 2: Growth rate (positive values of $\omega^{*}$ ) against wave number $k^{*}$ for three values of parameter $L_{\rho}^{*}=0.0,2.0,5.0$, keeping the other parameters fixed $\lambda^{*}=1.0, L_{T}^{*}=1.0, v^{*}=1.0$.

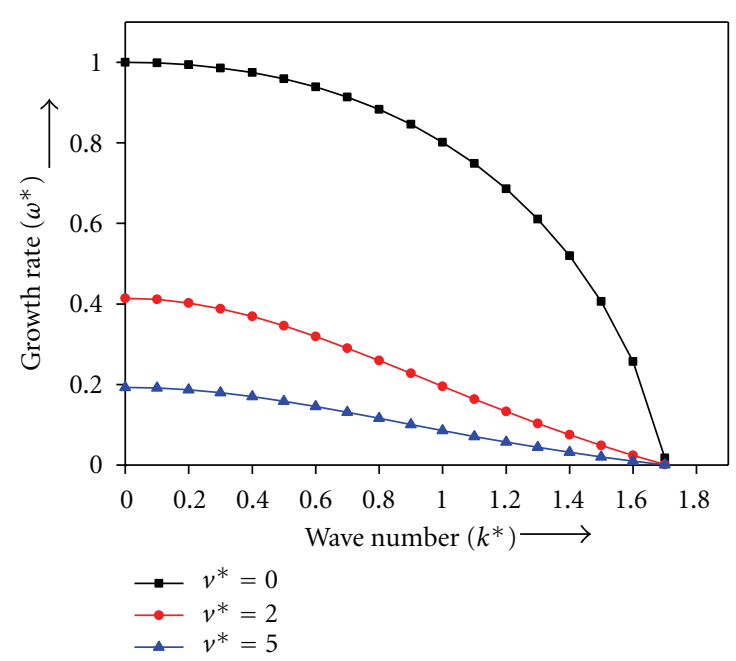

FIGURE 3: Growth rate (positive values of $\omega^{*}$ ) against wave number $k^{*}$ for three values of parameter $v^{*}=0.0,2.0,5.0$, keeping the other parameters fixed $L_{\rho}^{*}=1.0, \lambda^{*}=1.0, L_{T}^{*}=1.0$.

is destabilizing. From Figure 3 we conclude that growth rate decreases with increasing viscosity of the medium. Thus the effect of viscosity is stabilizing.

To discuss the stability of the system, if constant term of cubic equation (11) is greater than zero, then all the coefficients of (11) must be positive. Equation (11) is a thirddegree equation in the power of $\omega$ having its coefficients positive, which is a necessary condition for the stability of the system. To achieve the sufficient condition the principal 
diagonal minors of Hurwitz matrix must be positive. The principal diagonal minors are

$$
\begin{gathered}
\Delta_{1}=\left\{v k^{2}+(\gamma-1)\left(\frac{T \rho L_{T}}{p}+\frac{\lambda k^{2} T}{p}\right)\right\}>0, \\
\Delta_{2}=\left\{v k^{2}\left[\Delta_{1}(\gamma-1)\left(\frac{T \rho L_{T}}{p}+\frac{\lambda k^{2} T}{p}\right)+c^{2} k^{2}-4 \pi G \rho\right]\right. \\
\left.+(\gamma-1) k^{2} \rho L_{\rho}\right\}>0, \\
\Delta_{3}=\Delta_{2}\left\{k^{2}(\gamma-1)\left(T L_{T}-\rho L_{\rho}+\frac{\lambda k^{2} T}{\rho}\right)\right. \\
\left.-4 \pi G \rho(\gamma-1)\left(\frac{T \rho L_{T}}{p}+\frac{\lambda k^{2} T}{p}\right)\right\}>0 .
\end{gathered}
$$

Since $\Omega_{j}^{2}>0, \Omega_{I}^{2}>0$ and $\gamma>1$, it is clear that all the $\Delta s$ are positive hence system represented by (11) is stable system.

Now we wish to examine the effect of radiative heat-loss function in the considered system with some simplifications, and at the same time we wish to investigate the physics involved in such simplifications in the present problem.

\subsubsection{For Viscous Medium}

\section{(1) Effect of Radiative Heat-Loss Function}

For nonradiative medium $\left(L_{T, \rho}=0, v=\lambda \neq 0\right)$ (11) becomes

$$
\begin{aligned}
& \omega^{3}+\left(v k^{2}+\frac{\gamma \lambda k^{2}}{\rho c_{p}}\right) \omega^{2}+\left(v k^{2} \frac{\gamma \lambda k^{2}}{\rho c_{p}}+c^{2} k^{2}-4 \pi G \rho\right) \omega \\
& +\frac{\gamma \lambda k^{2}}{\rho c_{p}}\left(c^{\prime 2} k^{2}-4 \pi G \rho\right)=0 .
\end{aligned}
$$

The condition of instability from constant term of (18) is $\left(c^{\prime 2} k^{2}-4 \pi G \rho\right)<0$, or

$$
k^{2}<\frac{4 \pi G \rho}{c^{\prime 2}}
$$

if

$$
k_{J_{2}}^{2}=\frac{4 \pi G \rho}{c^{\prime 2}}, \quad \text { or } \quad \lambda_{J 2}=c^{\prime}\left[\frac{\pi}{G \rho}\right]^{1 / 2} \text {. }
$$

The medium is unstable for wave length $\lambda>\lambda_{J 2}$, where $\lambda_{J 2}$ is the modified Jeans wave length for thermally conducting medium. On comparing (18) and (11) we see that there is no new mode coming due to inclusion of radiative heat-loss functions, but the condition of instability gets modified in form of (11). Also it is interesting to note that the thermal conductivity term is separated from condition of instability when we neglect the effect of heatloss function in (11).
For nonthermal and nonradiating medium $\left(\lambda=L_{T, \rho}=\right.$ $0, v \neq 0)$ the condition of instability obtained from (11) is

$$
\begin{gathered}
\left(c^{2} k^{2}-4 \pi G \rho\right)<0, \\
\lambda_{J}=c\left[\frac{\pi}{G \rho}\right]^{1 / 2} .
\end{gathered}
$$

The medium is unstable for wave length $\lambda>\lambda_{J}$, where $\lambda_{J}$ is the Jeans wave length. The condition of Jeans instability given by (21) is identical to Chandrasekhar [5]. On comparing (12) and (21) it is clear that due to the presence of radiative heat-loss functions and thermal conductivity the fundamental criterion of Jeans gravitational instability changes into radiative instability criterion. Also, we conclude that one mode is increased because of inclusion of radiative heat-loss functions.

\subsubsection{For Nonviscous Medium}

(1) Effect of Radiative Heat-Loss Function

For inviscid and nonradiating medium $\left(v=L_{T, \rho}=0\right.$, $\lambda \neq 0$ ) (11) becomes

$$
\begin{gathered}
\omega^{3}+\frac{\gamma \lambda k^{2}}{\rho c_{p}} \omega^{2}+\left(c^{2} k^{2}-4 \pi G \rho\right) \omega \\
+\frac{\gamma \lambda k^{2}}{\rho c_{p}}\left(c^{\prime 2} k^{2}-4 \pi G \rho\right)=0 .
\end{gathered}
$$

The condition of instability is $\left(c^{\prime 2} k^{2}-4 \pi G \rho\right)<0$, it is discussed in (19) and (20). On comparing (23) and (11) we see that there is no new mode which comes due to inclusion of radiative heat-loss functions, but the condition of instability gets modified in form of (12). In both the cases whether the medium is viscous or nonviscous, the condition of instability is the same; hence, we conclude that viscosity of the medium has no effect on the condition of instability, and it only modifies the growth rate of the instability for viscous case.

For inviscid, thermally nonconducting and nonradiating medium $\left(v=\lambda=L_{T, \rho}=0\right)$ the condition of instability obtained from $(11)$ is $\left(c^{2} k^{2}-4 \pi G \rho\right)<0$, and it is discussed in (21) and (22). We see that inclusion of viscosity cannot change the condition of instability, but it modifies the growth rate of instability.

Thus we conclude that for longitudinal wave propagation as given by (8) the system is unstable only for modified Jeans condition, and else it is stable. Also the modified Jeans criterion remains unaffected by FLR corrections, viscosity, magnetic field, and finite electrical resistivity, but radiative heat-loss function and thermal conductivity modifies the Jeans expression and the fundamental Jeans instability criterion becomes radiative instability criterion. The growth rate of the system is modified by the presence of radiative heat-loss functions, thermal conductivity, and viscosity. The growth rate of Alfven mode is modified by the presence of viscosity, finite electrical resistivity, and FLR corrections. 
From the curves we find that temperature-dependent heatloss function and viscosity have stabilizing influence, whereas density-dependent heat-loss function has a destabilizing influence on the self-gravitational instability of plasma.
4.2. Transverse Propagation $\left(k_{x}=k, k_{z}=0\right)$. For this case, we assume that all the perturbations are transverse to the direction of magnetic field (i.e., $k_{x}=k, k_{z}=0$ ). The dispersion relation $(7)$ reduces to

$$
\begin{aligned}
\left(\omega+v k^{2}\right)^{2}\left\{\omega v_{0}^{2} k^{4}+\left(\omega+v k^{2}\right)\right. & {\left[\omega^{2}+\omega v k^{2}+\frac{\omega V^{2} k^{2}}{\omega+\eta k^{2}}+\frac{\omega\left(c^{2} k^{2}-4 \pi G \rho\right)}{\omega+(\gamma-1)\left(T \rho L_{T} / p+\lambda k^{2} T / p\right)}\right.} \\
& \left.\left.+\frac{(\gamma-1)\left\{k^{2}\left(T L_{T}-\rho L_{\rho}+\lambda k^{2} T / p\right)-4 \pi G \rho\left(T \rho L_{T} / p+\lambda k^{2} T / p\right)\right\}}{\omega+(\gamma-1)\left(T \rho L_{T} / p+\lambda k^{2} T / p\right)}\right]\right\}=0 .
\end{aligned}
$$

We find that for transverse mode of propagation the dispersion relation is modified due to the presence of FLR corrections, radiative heat-loss function, thermal conductivity, viscosity, finite electrical resistivity, and magnetic field. The dispersion relation (24) has two different components. The first component of the dispersion relation (24) represents a damped mode modified by the presence of viscosity of the medium and is discussed in (9).

The second component of the dispersion relation (24) on simplifying gives

$$
\begin{aligned}
& \omega^{5}+\left[2 v k^{2}+\eta k^{2}+(\gamma-1)\left(\frac{T \rho L_{T}}{p}+\frac{\lambda k^{2} T}{p}\right)\right] \omega^{4} \\
& +\left\{2 v k^{2}\left[\eta k^{2}+(\gamma-1)\left(\frac{T \rho L_{T}}{p}+\frac{\lambda k^{2} T}{p}\right)\right]\right. \\
& +v^{2} k^{4}+\eta k^{2}(\gamma-1)\left(\frac{T \rho L_{T}}{p}+\frac{\lambda k^{2} T}{p}\right) \\
& \left.+V^{2} k^{2}+v_{0}^{2} k^{4}+c^{2} k^{2}-4 \pi G \rho\right\} \omega^{3} \\
& +\left\{2 \eta k^{2} v k^{2}(\gamma-1) \times\left(\frac{T \rho L_{T}}{p}+\frac{\lambda k^{2} T}{p}\right)\right. \\
& +\left[\eta k^{2}+(\gamma-1)\left(\frac{T \rho L_{T}}{p}+\frac{\lambda k^{2} T}{p}\right)\right]\left(v^{2} k^{4}+v_{0}^{2} k^{4}\right) \\
& +V^{2} k^{2}\left[v k^{2}+(\gamma-1) \times\left(\frac{T \rho L_{T}}{p}+\frac{\lambda k^{2} T}{p}\right)\right] \\
& +\left(c^{2} k^{2}-4 \pi G \rho\right)\left(v k^{2}+\eta k^{2}\right) \\
& +(\gamma-1)\left[k^{2}\left(T L_{T}-\rho L_{\rho}+\frac{\lambda k^{2} T}{\rho}\right)-4 \pi G \rho\right. \\
& \left.\left.\times\left(\frac{T \rho L_{T}}{p}+\frac{\lambda k^{2} T}{p}\right)\right]\right\} \omega^{2}
\end{aligned}
$$

$$
\begin{aligned}
+\left\{v k ^ { 2 } \left[\eta k^{2} v k^{2}(\gamma-1)\left(\frac{T \rho L_{T}}{p}+\frac{\lambda k^{2} T}{p}\right)\right.\right. \\
+V^{2} k^{2}(\gamma-1) \\
\left.\quad \times\left(\frac{T \rho L_{T}}{p}+\frac{\lambda k^{2} T}{p}\right)+\eta k^{2}\left(c^{2} k^{2}-4 \pi G \rho\right)\right] \\
+\eta k^{2} v_{0}^{2} k^{4}(\gamma-1)\left(\frac{T \rho L_{T}}{p}+\frac{\lambda k^{2} T}{p}\right) \\
+(\gamma-1)\left[k^{2}\left(T L_{T}-\rho L_{\rho}+\frac{\lambda k^{2} T}{\rho}\right)\right. \\
\left.\left.-4 \pi G \rho\left(\frac{T \rho L_{T}}{p}+\frac{\lambda k^{2} T}{p}\right)\right]\left(v k^{2}+\eta k^{2}\right)\right\} \omega
\end{aligned}
$$$$
+\eta k^{2} v k^{2}
$$$$
\times\left[k^{2}(\gamma-1)\left(T L_{T}-\rho L_{\rho}+\frac{\lambda k^{2} T}{\rho}\right)\right.
$$$$
\left.-4 \pi G \rho(\gamma-1)\left(\frac{T \rho L_{T}}{p}+\frac{\lambda k^{2} T}{p}\right)\right]=0 .
$$

The above fifth degree equation in $\omega$ represents the combined influence of FLR corrections, radiative heat-loss function, thermal conductivity, finite electrical resistivity, viscosity, and magnetic field on self-gravitational instability of plasma. When constant term of (25) is less than zero, this allows at least one positive real root which corresponds to the instability of the system. The condition of instability obtained from constant term of (25) is given as

$$
\begin{aligned}
(\gamma-1)\left[k^{2}\left(T L_{T}-\rho L_{\rho}+\frac{\lambda k^{2} T}{\rho}\right)\right. & \\
& \left.-4 \pi G \rho\left(\frac{T \rho L_{T}}{p}+\frac{\lambda k^{2} T}{p}\right)\right]<0 .
\end{aligned}
$$

The above condition of instability is independent of FLR correction, finite electrical resistivity, viscosity, and magnetic field strength and is discussed in (12). Thus from (25), we 
find that the modified Jeans condition of instability remains the same whether we consider the effect of FLR corrections or not, but, owing to the presence of FLR corrections, the growth rate of radiative instability is modified. This result can be easily explained by graphical representation as shown in Figure 6; by increasing values of FLR, we see that the growth rate of radiative instability decreases. Hence FLR shows stabilizing influence on the growth rate of radiative instability.

In several astrophysical plasma situations, such as in interstellar molecular clouds the formation of objects is mainly due to the unstable modes. Thus to discuss the effect of each parameter on the growth rate of unstable modes, we solve (25) numerically by using the relation given in (14) with the following additional dimensionless quantities:

$$
\begin{gathered}
V^{* 2}=\frac{V^{2}}{c^{2}}, \quad \eta^{*}=\frac{\eta(4 \pi G \rho)^{1 / 2}}{c^{2}}, \\
v_{0}^{*}=\frac{v_{0}(4 \pi G \rho)^{1 / 2}}{c^{2}} .
\end{gathered}
$$

For calculations we take the numerical values corresponding to the conditions in interstellar molecular clouds which are shown in (16), and the rest of values are taken as nondimensional. Numerical calculations were performed to determine the roots of $\omega^{*}$ as a function of wave number $k^{*}$ for several values of the different parameters involved taking $\gamma=5 / 3$. Out of the five modes, only one mode is unstable for which the calculations are presented in Figures $4-6$, where the growth rate $\omega^{*}$ (positive real value of $\omega$, after multiplying by $10^{16}$ ) has been plotted against the wave number $k^{*}$ (after multiplying by $10^{20}$ ) to show the dependence of the growth rate on the different physical parameters such as temperature-dependent heat-loss function $L_{T}^{*}$ (after multiplying by $10^{8}$ ), density-dependent heat-loss function $L_{\rho}^{*}$ (after multiplying by $10^{8}$ ), viscosity $v^{*}$ (after multiplying by $10^{24}$ ), and FLR corrections $v_{0}^{*}$ (after multiplying by $10^{24}$ ).

It is clear from Figure 4 that growth rate decreases with increasing temperature-dependent heat-loss function. Thus the effect of temperature-dependent heat-loss function is stabilizing. From Figure 5 we conclude that growth rate increases with increasing density-dependent heat-loss function. Thus the effect of density-dependent heat-loss function is destabilizing. One can observe from Figure 6 that the growth rate decreases with increasing FLR corrections. Thus the effect of FLR corrections is stabilizing. If the viscous effect of plasma is removed, then the stabilizing rate of FLR corrections increases and the peak value of the curve also increases. Hence we conclude that both FLR corrections and viscosity stabilize the system.

To discuss the stability of the system given by dispersion relation (25), if constant term of equation (25) is greater than zero, then all the coefficients of the equation (25) must be positive. Equation (25) is a fifth degree equation in the power of $\omega$; hence, it will involve a lengthy algebra to calculate the principal diagonal minors of Hurwitz matrix. Since (25) has positive real coefficients, having $\Omega_{j}^{2}>0, \Omega_{I}^{2}>0$ and $\gamma>1$, so it is sufficient to show that alternate $\Delta s$ are positive; hence, system represented by (25) is a stable system.

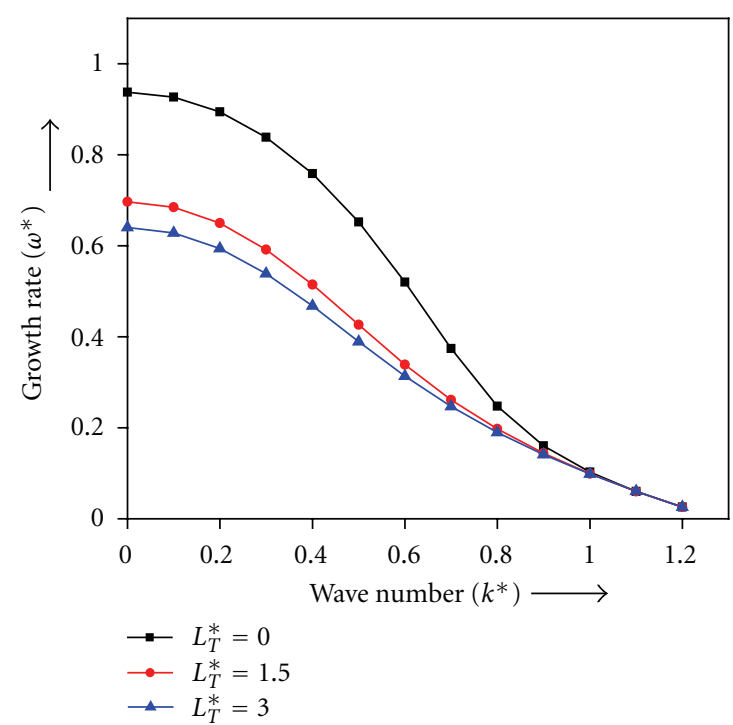

FIGURE 4: Growth rate (positive values of $\omega^{*}$ ) against wave number $k^{*}$ for three values of parameter $L_{T}^{*}=0.0,1.5,3.0$, keeping the other parameters fixed $v^{*}=0.5, L_{\rho}^{*}=0.0, \lambda^{*}=1.0, v_{0}^{*}=1.0, V^{*}=$ $1.0, \eta^{*}=0.5$.

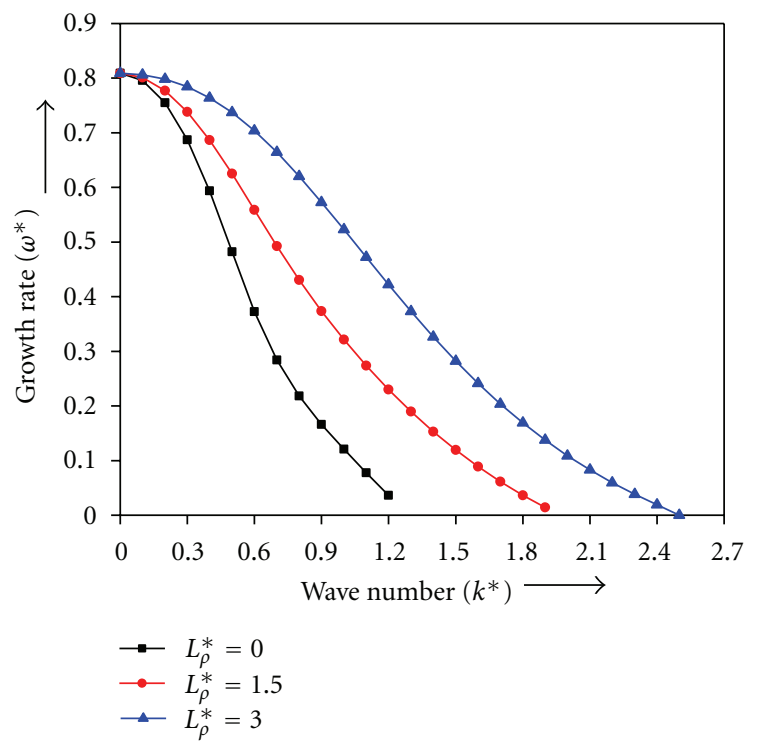

Figure 5: Growth rate (positive values of $\omega^{*}$ ) against wave number $k^{*}$ for three values of parameter $L_{\rho}^{*}=0.0,1.5,3.0$, keeping the other parameters fixed $v_{0}^{*}=1.0, \lambda^{*}=1.0, v^{*}=0.5, V^{*}=1.0, \eta^{*}=$ $0.5, L_{T}^{*}=0.5$.

Now we wish to examine the effect of FLR corrections and radiative heat-loss function on the considered system with some simplifications such as viscous and nonviscous medium, and at the same time we wish to investigate the physics involved in such simplifications in the present problem. 


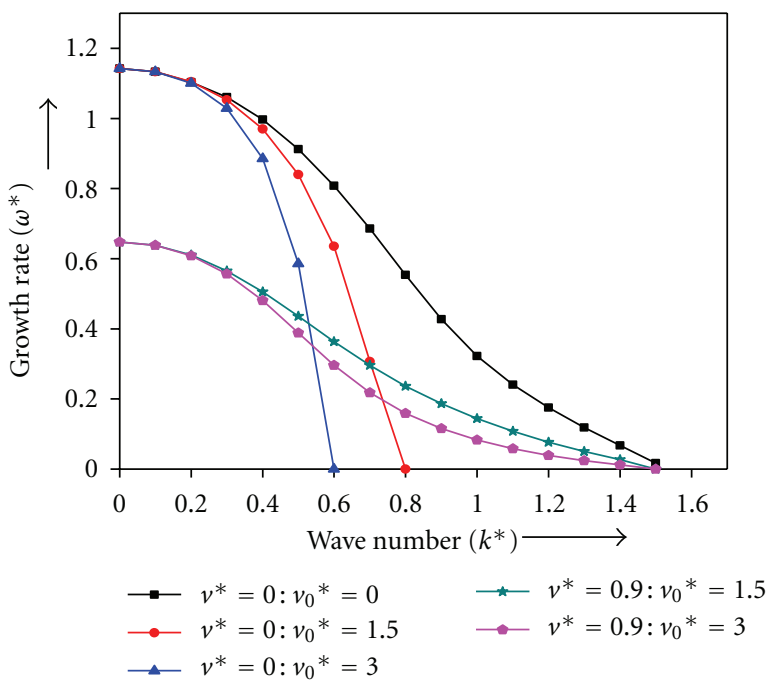

Figure 6: Growth rate (positive values of $\omega^{*}$ ) against wave number $k^{*}$ for several values of parameter $v_{0}^{*}$ keeping the other parameters fixed $L_{\rho}^{*}=0.5, L_{T}^{*}=0.5, \lambda^{*}=1.0, V^{*}=1.0, \eta^{*}=0.5 . v^{*}=0.0$ : (1) $v_{0}^{*}=0.0,(2) v_{0}^{*}=1.5(3) v_{0}^{*}=3.0 . v^{*}=0.9$ : (4) $v_{0}^{*}=1.5,(5)$ $v_{0}^{*}=3.0$.

\subsubsection{For Viscous Medium}

(1) Effect of Radiative Heat-Loss Function with FLR Corrections

For nonradiative medium, but in the presence of FLR corrections $\left(L_{T, \rho}=0, v=\lambda=V=\eta=v_{0} \neq 0\right)$ the condition of instability obtained from the constant term of (25) is given as

$$
\left(c^{\prime 2} k^{2}-4 \pi G \rho\right)<0 .
$$

The above (28) is identical to that of Vaghela and Chhajlani [19]. We see that there is no new mode due to inclusion of radiative heat-loss function, but the growth rate of instability gets modified due to inclusion of radiative heatloss functions and FLR corrections. Also it is interesting to note that, on neglecting the effect of radiative heat-loss function in (25), the thermal conductivity parameter (i.e., $\lambda$ ) is separated from instability condition as shown in (28). So we conclude that on neglecting the effect of radiative heat-loss function the condition of instability is independent of both the radiative heat-loss function and thermal conductivity of the medium, but the growth rate of instability is affected by thermal conductivity and FLR corrections.

For nonradiative and nonthermal medium, but in presence of FLR corrections $\left(L_{T, \rho}=\lambda=0, v=V=\eta=v_{0} \neq 0\right)$ the condition of instability obtained from (25) is given as

$$
\left(c^{2} k^{2}-4 \pi G \rho\right)<0 .
$$

We find that one mode is increased due to inclusion of radiative heat-loss functions. On comparing (26) and (29), it is clear that owing to the presence of thermal conductivity and radiative heat-loss functions the fundamental criterion of Jeans gravitational instability changes into radiative instability criterion. In case of transverse mode of propagation, for viscous medium, FLR corrections have no effect on the condition of instability for radiating or nonradiating medium, but the growth rate of instability is modified by FLR corrections; that is, FLR stabilizes the system in all the cases. The joint stabilizing effect of FLR corrections and viscosity decreases the peak value of growth rate of the system shown in Figure 6.

\subsubsection{For Nonviscous Medium}

\section{(1) Effect of FLR Corrections}

For inviscid medium, but in presence of FLR corrections $\left(v=0, \eta=L_{T, \rho}=V=\lambda=v_{0} \neq 0\right)(25)$ becomes

$$
\begin{gathered}
\omega^{4}+\left[\eta k^{2}+(\gamma-1)\left(\frac{T \rho L_{T}}{p}+\frac{\lambda k^{2} T}{p}\right)\right] \omega^{3} \\
+\left[\eta k^{2}(\gamma-1)\left(\frac{T \rho L_{T}}{p}+\frac{\lambda k^{2} T}{p}\right)+V^{2} k^{2}\right. \\
\left.+v_{0}^{2} k^{4}+c^{2} k^{2}-4 \pi G \rho\right] \omega^{2} \\
+\left\{v_{0}^{2} k^{4}\left[\eta k^{2}+(\gamma-1)\left(\frac{T \rho L_{T}}{p}+\frac{\lambda k^{2} T}{p}\right)\right]\right. \\
+V^{2} k^{2}(\gamma-1) \\
\times\left(\frac{T \rho L_{T}}{p}+\frac{\lambda k^{2} T}{p}\right)+\eta k^{2}\left(c^{2} k^{2}-4 \pi G \rho\right) \\
+\left[k^{2}(\gamma-1)\left(T L_{T}-\rho L_{\rho}+\frac{\lambda k^{2} T}{\rho}\right)\right. \\
+\eta k^{2}\left\{v_{0}^{2} k^{4}(\gamma-1)\left(\frac{T \rho L_{T}}{p}+\frac{\lambda k^{2} T}{p}\right)+k^{2}(\gamma-1)\right. \\
\quad \times\left(T L_{T}-\rho L_{\rho}+\frac{\lambda k^{2} T}{\rho}\right) \\
\left.\left.-4 \pi G \rho(\gamma-1)\left(\frac{T \rho L_{T}}{p}+\frac{\lambda k^{2} T}{p}\right)\right]\right\}
\end{gathered}
$$

The above equation (30) is a modified form of Chhajlani and Parihar [20] by inclusion of radiative heat-loss function, thermal conductivity, and finite electrical resistivity. The condition of instability obtained from constant term of (30) is given as

$$
\begin{aligned}
& \left\{k^{2}\left(T L_{T}-\rho L_{\rho}+\frac{\lambda k^{2} T}{\rho}\right)\right. \\
& \left.\quad-\left(4 \pi G \rho-v_{0}^{2} k^{4}\right)\left(\frac{T \rho L_{T}}{p}+\frac{\lambda k^{2} T}{p}\right)\right\}<0 .
\end{aligned}
$$


From the above condition of instability given by (31) we conclude that FLR correction tries to stabilize the radiative instability. Also on comparing (25) and (30) we see that inclusion of viscosity removes the effects of FLR correction from condition of instability. So in both the cases either the system is viscous or nonviscous, FLR correction stabilizes the growth rate of radiative instability, as shown in Figure 6. Thus in presence of FLR correction the condition of instability given by Bora and Talwar [34] is modified as given by (31). In absence of FLR corrections (30) is identical to Bora and Talwar [34]; thus, the dispersion relation is modified due to the inclusion of effects of FLR in the present system. Thus we find that due to the presence of FLR corrections Jeans criterion of instability is modified, and also the growth rate of instability in this case is modified with the inclusion of FLR correction in the present MHD set of equations. The above condition of instability (31) can be written in nondimensional form as

$$
\left\{\left(\lambda^{*} k^{*^{2}}+L_{T}^{*}\right)\left[v_{0}^{*^{2}} k^{*^{4}}+\frac{k^{*^{2}}}{\gamma}-1\right]-k^{*^{2}} L_{\rho}^{*}\right\}<0 .
$$

In absence of FLR corrections the above condition of instability is the same as given by Bora and Talwar [34] and by Hunter [25] for an electrically nonconducting gas. We conclude that condition of instability given by Bora and Talwar [34] is modified by inclusion of FLR corrections in our case. Also the condition of instability given by (31) is the modified form of Field [24] by inclusion of FLR correction and self-gravitation. Thus the present result is the improvement of Field [24], Hunter [25], and Bora and Talwar [34]. Condition (32) involves the derivatives $L_{T}^{*}$ and $L_{\rho}^{*}$ of the heat-loss function with respect of local temperature and density, and $v_{0}^{*}$ is FLR correction factor in the system. It may be noted that for a density-independent heat-loss function $\left(L_{\rho}^{*}=0\right)$, which increases with temperature $\left(L_{T}^{*}>\right.$ $0)$, condition (32) gives instability if $k^{*^{2}}<\left(\gamma-1 / \gamma v_{0}^{* 2}\right)$. However if instead, the heat-loss function decreases with temperature $\left(L_{T}^{*}<0\right)$, and the instability comes to $k^{* 2}$ lying between $\left(\gamma-1 / \gamma v_{0}^{* 2}\right)$ and $\left\{\left|L_{T}^{*}\right| / \lambda^{*}\right\}$.

For a purely density-dependent heat-loss function $\left(L_{T}^{*}=\right.$ 0 ), condition (32) gives instability if

$$
k^{*^{2}}<\frac{1}{2}\left\{ \pm\left[\left(\frac{1}{\gamma v_{0}^{* 2}}\right)^{2}+\frac{4}{v_{0}^{* 2}}\left(1+\frac{L_{\rho}^{*}}{\lambda^{*}}\right)\right]^{1 / 2}-\left(\frac{1}{\gamma v_{0}^{* 2}}\right)\right\} .
$$

The above equation (33) applies for $L_{\rho}^{*}>0$ or $\left|L_{\rho}^{*}\right|<\lambda^{*}$ if the heat-loss function, respectively, increases or decreases with increase in density.

(2) Effect of Radiative Heat-Loss Function with FLR Corrections

For nonradiating and inviscid medium, but in presence of $\operatorname{FLR}\left(L_{T, \rho}=v=0, \eta=\lambda=v_{0}=V \neq 0\right)$ the condition of instability obtained from (25) is given as

$$
k^{2}\left(c^{\prime 2}+v_{0}^{2} k^{2}\right)<4 \pi G \rho .
$$

The critical Jeans wave number and critical Jeans wave length are given as

$$
k_{J 3}^{2}=\frac{\left\{-c^{\prime 2} \pm\left(c^{4}+16 \pi G \rho v_{0}^{2}\right)^{1 / 2}\right\}}{2 v_{0}^{2}} .
$$

Or

$$
\lambda_{J 3}=\left[\frac{8 \pi^{2} v_{0}^{2}}{\left\{-c^{\prime 2} \pm\left(c^{\prime 4}+16 \pi G \rho v_{0}^{2}\right)^{1 / 2}\right\}}\right]^{1 / 2} .
$$

Condition (34) is identical of Vaghela and Chhajlani [19] for $(\varepsilon=1)$ in their case. From (34) we conclude that FLR have stabilizing effect on the instability of the system. We find that one mode is increased due to inclusion of viscosity, and we conclude that inclusion of viscosity removes the effect of FLR corrections from the condition of instability. Also on comparing (31) and (34), we conclude that condition of instability given by Vaghela and Chhajlani [19] is modified by inclusion of radiative effects and the growth rate of instability given by (31) is modified by radiative heat-loss function and FLR corrections.

For inviscid, nonradiating and thermally nonconducting medium, but in presence of FLR $\left(v=L_{T, \rho}=\lambda=0, \eta=\right.$ $\left.v_{0} \neq 0\right)$, the condition of instability obtained from (25) is given as

$$
\left\{\left(c^{2}+v_{0}^{2} k^{2}\right) k^{2}-4 \pi G \rho\right\}<0 .
$$

The critical Jeans wave number or critical Jeans wave length is given as

$$
k_{J 4}^{2}=\frac{\left\{-c^{2} \pm\left(c^{4}+16 \pi G \rho v_{0}^{2}\right)^{1 / 2}\right\}}{2 v_{0}^{2}} .
$$

Or

$$
\lambda_{J 4}=\left[\frac{8 \pi^{2} v_{0}^{2}}{\left\{-c^{2} \pm\left(c^{4}+16 \pi G \rho v_{0}^{2}\right)^{1 / 2}\right\}}\right]^{1 / 2} .
$$

From (37) we find that Jeans condition of gravitational instability gets modified by FLR corrections. On comparing (37) and (31) we conclude that inclusion of thermal conductivity and radiative heat-loss function modifies both the Jeans condition of instability and growth rate of instability in presence of FLR corrections. Hence the fundamental Jeans criterion of gravitational instability gets modified into radiative instability criterion by inclusion of thermal conductivity and radiative heat-loss functions in the presence of FLR corrections.

(3) Effect of Electrical Conductivity with FLR Corrections

For inviscid and infinitely conducting medium, but in presence of FLR $\left(v=\eta=0, L_{T, \rho}=V=\lambda=v_{0} \neq 0\right)$, the 
condition of instability obtained from constant term of (25) is given as

$$
\begin{aligned}
& \left\{k^{2}\left(T L_{T}-\rho L_{\rho}+\frac{\lambda k^{2} T}{\rho}\right)\right. \\
& \left.\quad-\left(4 \pi G \rho-v_{0}^{2} k^{4}-V^{2} k^{2}\right)\left(\frac{T \rho L_{T}}{p}+\frac{\lambda k^{2} T}{p}\right)\right\}<0 .
\end{aligned}
$$

From the above condition of instability given by (40) we conclude that FLR correction and magnetic field strength try to stabilize the radiative instability. Also on comparing (26) and (40) we see that inclusion of viscosity and finite conductivity removes the effect of FLR correction and magnetic field from condition of instability. So in both the cases whether the system is finitely conducting or infinitely conducting magnetic field and FLR correction stabilizes the growth rate of radiative instability. The above condition of instability is identical to Kaothekar and Chhajlani [36]. Kaothekar and Chhajlani [36] have considered the effect of permeability, but, in the present problem, we have not considered the effect of permeability, so we conclude that the conditions of instability for permeable or nonpermeable medium are same, but the growth rate of the system is modified by the presence of permeability. The above condition of instability (40) can be written in nondimensional form as

$$
\begin{aligned}
& \left\{\left(\lambda^{*} k^{* 2}+L_{T}^{*}\right)\left[v_{0}^{* 2} k^{* 4}+k^{* 2}\left(\frac{1}{\gamma}+V^{* 2}\right)-1\right]\right. \\
& \left.-k^{* 2} L_{\rho}^{*}\right\}<0 .
\end{aligned}
$$

In absence of FLR corrections the above condition of instability is identical to Bora and Talwar [34] excluding electron inertia in that case. We conclude that condition of instability given by Bora and Talwar [34] is modified by inclusion of FLR corrections in our case. Thus the present results are the improvement of Bora and Talwar [34] due to FLR corrections.

From condition of instability (41) it may be noted that for a density-independent heat-loss function $\left(L_{\rho}^{*}=0\right)$, which increases with temperature $\left(L_{T}^{*}>0\right)$, it gives instability if $k^{*^{2}}<\left(1 / v_{0}^{* 2}\right)\left\{1-\left[(1 / \gamma)+V^{* 2}\right]\right\}$. However if instead, the heat-loss function decreases with temperature $\left(L_{T}^{*}<0\right)$, the instability comes to $k^{* 2}$ lying between $\left(1 / v_{0}^{* 2}\right)\{1-[(1 / \gamma)+$ $\left.\left.V^{* 2}\right]\right\}$ and $\left\{\left|L_{T}^{*}\right| / \lambda^{*}\right\}$.

For a purely density-dependent heat-loss function $\left(L_{T}^{*}=\right.$ $0)$, condition (41) gives instability if

$$
\begin{aligned}
k^{* 2}<\frac{1}{2}\{ \pm & {\left[\frac{1}{v_{0}^{* 4}}\left(\frac{1}{\gamma}+V^{*^{2}}\right)^{2}+\frac{4}{v_{0}^{* 2}}\left(1+\frac{L_{\rho}^{*}}{\lambda^{*}}\right)\right]^{1 / 2} } \\
& \left.-\frac{1}{v_{0}^{* 2}}\left(\frac{1}{\gamma}+V^{* 2}\right)\right\} .
\end{aligned}
$$

The above equation (42) applies for $L_{\rho}^{*}>0$ or $\left|L_{\rho}^{*}\right|<\lambda^{*}$, if the heat-loss function, respectively, increases or decreases with increase in density.

\section{(4) Effect of Nongravitation and FLR Corrections}

For inviscid, nongravitating, infinitely conducting, radiating, thermally conducting, magnetized medium with FLR corrections $\left(v=\eta=G=0, v_{0}=V=L_{T, \rho}=\lambda \neq 0\right)(25)$ becomes

$$
\begin{aligned}
\omega^{3}+ & {\left[(\gamma-1)\left(\frac{T \rho L_{T}}{p}+\frac{\lambda k^{2} T}{p}\right)\right] \omega^{2} } \\
+ & {\left[V^{2} k^{2}+v_{0}^{2} k^{4}+c^{2} k^{2}\right] \omega } \\
+ & (\gamma-1) k^{2}\left[\left(V^{2}+v_{0}^{2} k^{2}\right)\right. \\
& \left.\times\left(\frac{T \rho L_{T}}{p}+\frac{\lambda k^{2} T}{p}\right)+\left(T L_{T}-\rho L_{\rho}+\frac{\lambda k^{2} T}{\rho}\right)\right]=0 .
\end{aligned}
$$

The condition of instability obtained from constant term of (43) is given as

$$
\begin{gathered}
{\left[\left(v_{0}^{2} k^{2}+V^{2}\right)\left(\frac{T \rho L_{T}}{p}+\frac{\lambda k^{2} T}{p}\right)\right.} \\
\left.+\left(T L_{T}+\frac{\lambda k^{2} T}{\rho}-\rho L_{\rho}\right)\right]<0 .
\end{gathered}
$$

From above equation (44) we see that FLR correction and magnetic field strength try to stabilize the system. If we neglect the effect of FLR and magnetic field the above dispersion relation (43) is identical to Field [24]. In the present case we have considered the effects of FLR correction and magnetic field, but Field [24] has not considered these effects. Thus in the present analysis both the dispersion relation and the condition of instability get modified due to the presence of FLR corrections and magnetic field strength. On comparing (40) and (44) we see that consideration of self-gravitation modifies the thermal instability criterion into radiative instability criterion. Also from (25) it is clear that the growth rate of the dispersion relation given by Field [24] is modified due to the presence of FLR correction, viscosity, magnetic field, finite electrical conductivity, and self-gravitation in our present case. Hence these are the new results in our present problem than that of Field [24].

Thus we conclude that for transverse wave propagation the Jeans criterion is affected by FLR corrections, radiative heat-loss functions, thermal conductivity, viscosity, magnetic field strength, and finite electrical resistivity. From curves we find that FLR corrections, temperature-dependent heat-loss function, and viscosity have stabilizing influence, whereas density-dependent heat-loss function has destabilizing influence on the self-gravitational instability of plasma.

\section{Conclusion}

We have dealt with the self-gravitation instability of an infinite homogeneous viscous electrically and thermally conducting fluid including the effects of FLR corrections and radiative heat-loss function for star formation. The general 
dispersion relation is obtained, which is modified due to the presence of considered physical parameters. This dispersion relation is reduced for longitudinal and transverse mode of propagation to the direction of the magnetic field. We find that Jeans criterion remains valid and gets modified because of FLR corrections, radiative heat-loss function, thermal conductivity, and magnetic field. We also find that the presence of thermal conductivity and radiative heatloss function modifies the fundamental Jeans criterion of gravitational instability into radiative instability criterion. The effect of viscosity parameter is found to stabilize the system in both the longitudinal and transverse mode of propagation. For longitudinal wave propagation magnetic field, viscosity, finite electrical resistivity, and FLR correction have no effect on Jeans criterion, but FLR corrections, viscosity, and finite electrical resistivity modify the growth rate of Alfven mode. For transverse wave propagation FLR corrections stabilize the growth rate of the system in all the cases, but it modifies the condition of instability only for the case of nonviscous medium. Also, magnetic field stabilizes the system but finite conductivity removes the effect of magnetic field there by destabilizing the system. Numerical calculation shows stabilizing effect of temperaturedependent heat-loss function, FLR corrections, and viscosity, where as destabilizing effect of density-dependent heat-loss function is on the self-gravitational instability.

\section{Acknowledgments}

The authors are highly thankful to Prof. and Head Dr. S. K. Ghosh, S. S. in Physics and Dr. T. R. Thapak, Hon'ble V. C. Vikram University Ujjain (MP) for their constant encouragements. S. Kaothekar is grateful to Er. Praveen Vashishtha, Chairman of Mahakal Institute of Technology for continuous support and guidance.

\section{References}

[1] C. Hayashi, "Evolution of protostars," Annual Review of Astronomy and Astrophysics, vol. 4, pp. 171-192, 1966.

[2] F. H. Shu, F. C. Adams, and S. Lizano, "Star formation in molecular clouds," Annual Review of Astronomy and Astrophysics, vol. 25, pp. 23-81, 1987.

[3] B. T. Draine and C. F. McKee, "Theory of interstellar shocks," Annual Review of Astronomy and Astrophysics, vol. 31, pp. 373432, 1993.

[4] C. F. McKee and E. C. Ostriker, "Theory of star formation," Annual Review of Astronomy and Astrophysics, vol. 45, pp. 565687, 2007.

[5] S. Chandrasekhar, Hydrodynamics and Hydromagnetic Stability, Clarendon Press, Oxford, UK, 1961.

[6] A. G. Pacholczyk and J. S. Stodolkiewicz, "On the gravitational instability of some magnetohydrodynamical systems of astrophysical interest," Acta Astronomica, vol. 10, pp. 1-29, 1960.

[7] N. K. Nayyar, "Magnetogravitational instability of an infinite medium with finite electrical and thermal conductivity," Zeitschrift fur Astrophysik, vol. 52, pp. 266-271, 1961.

[8] S. Shaikh, A. Khan, and P. K. Bhatia, "Stability of thermally conducting plasma in a variable magnetic field," Astrophysics and Space Science, vol. 312, pp. 35-40, 2007.
[9] C.-C. Yang, R. A. Gruendl, Y.-H. Chu, M.-M. Mac Low, and Y. Fukui, "Large-scale gravitational instability and star formation in the large magellanic cloud," The Astrophysical Journal, vol. 671, pp. 374-379, 2007.

[10] A. C. Borah and A. K. Sen, "Gravitational instability of partially ionized molecular clouds," Journal of Plasma Physics, vol. 73, no. 6, pp. 831-838, 2007.

[11] K. Avinash, B. Eliasson, and P. K. Shukla, "Dynamics of selfgravitating dust clouds and the formation of planetesimals," Physics Letters, Section A, vol. 353, pp. 105-108, 2006.

[12] K. V. Roberts and J. B. Taylor, "Magnetohydrodynamic equations for finite Larmor radius," Physical Review Letters, vol. 8, pp. 197-198, 1962.

[13] A. Jeffery and T. Taniuti, MHD Stability and Thermonuclear Containment, Academic Press, New York, NY, USA, 1966.

[14] J. D. Jukes, "Gravitational resistive instabilities in plasma with finite Larmor radius," Physics of Fluids, vol. 7, no. 1, pp. 52-58, 1964.

[15] L. V. Vandakurov, "On the theory of stability of a plasma in a strong longitudinal magnetic field, variable along the axis of symmetry," Journal of Applied Mathematics and Mechanics, vol. 28, no. 1, pp. 77-89, 1964.

[16] R. C. Sharma, "Gravitational instability of rotating plasma," Astrophysics and Space Science, vol. 29, pp. L1-L4, 1974.

[17] P. K. Bhatia and R. P. S. Chhonkar, "Larmor radius effects on the instability of a rotating layer of a self-gravitating plasma," Astrophysics and Space Science, vol. 115, no. 2, pp. 327-344, 1985.

[18] F. Herrnegger, "Effect of collisions and gyroviscosity on gravitational instability in a two-component plasma," Journal of Plasma Physics, vol. 8, pp. 393-400, 1972.

[19] D. S. Vaghela and R. K. Chhajlani, "Magnetogravitational stability of resistive plasma through porous medium with thermal conduction and FLR corrections," Contributions to Plasma Physics, vol. 29, pp. 77-89, 1989.

[20] R. K. Chhajlani and A. K. Parihar, "Magnetogravitational instability of anisotropic plasma with finite ion Larmor and generalized polytrope law," Contributions to Plasma Physics, vol. 34, pp. 669-681, 1994.

[21] N. M. Ferraro, "Finite Larmor radius effects on the magnetorotational instability," The Astrophysical Journal, vol. 662, pp. 512-516, 2007.

[22] I. Sandberg, H. Isliker, and V. P. Pavlenko, "Finite Larmor radius effects on the coupled trapped electron and ion temperature gradient modes," Physics of Plasmas, vol. 14, Article ID 092504, 2007.

[23] E. Devlen and E. R. Pekünlü, "Finite Larmor radius effects on weakly magnetized, dilute plasmas," Monthly Notices of the Royal Astronomical Society, vol. 404, pp. 830-836, 2010.

[24] G. B. Field, "Thermal instability," The Astrophysical Journal, vol. 142, pp. 531-567, 1965.

[25] J. H. Hunter, "The role of thermal instabilities in star formation," Monthly Notices of the Royal Astronomical Society, vol. 133, pp. 239-245, 1966.

[26] M. H. Ibanez S., "Sound and thermal waves in a fluid with an arbitrary heat-loss function," The Astrophysical Journal, vol. 290, pp. 33-46, 1985.

[27] W.-T. Kim and R. Narayan, "Thermal instability in clusters of galaxies with conduction,” The Astrophysical Journal, vol. 596, pp. 889-902, 2003.

[28] A. E. Radwan, "Variable streams self-gravitating instability of radiating rotating gas cloud," Applied Mathematics and Computation, vol. 148, no. 2, pp. 331-339, 2004. 
[29] K. Menou, S. A. Balbus, and H. C. Spruit, "Local axisymmetric diffusive stability of weakly magnetized, differentially rotating, stratified fluids," The Astrophysical Journal, vol. 607, no. 1, pp. 564-574, 2004.

[30] S. I. Inutsuka, H. Koyama, and T. Inoue, "The role of thermal instability in interstellar medium," in Proceedings of the AIP Conference on Magnetic Fields in the Universe: from Laboratory and Stars to Primordial Structures, vol. 784, pp. 318-328, Angra dos Reis, Brazil, December 2004.

[31] M. Shadmehri and S. Dib, "Magnetothermal condensation modes including the effects of charged dust particles," Monthly Notices of the Royal Astronomical Society, vol. 395, pp. 985-990, 2009.

[32] S. Shaikh, A. Khan, and P. K. Bhatia, “Jeans' gravitational instability of a thermally conducting plasma," Physics Letters, Section A, vol. 372, no. 9, pp. 1451-1457, 2008.

[33] M. Aggarwal and S. P. Talwar, "Magnetothermal instability in a rotating gravitating fluid," Monthly Notices of the Royal Astronomical Society, vol. 146, pp. 235-242, 1969.

[34] M. P. Bora and S. P. Talwar, "Magnetothermal instability with generalized Ohm's law," Physics of Fluids B, vol. 5, no. 3, pp. 950-955, 1993.

[35] M. F. El-Sayed and R. A. Mohamed, "Gravitational instability of rotating viscoelastic partially ionized plasma in the presence of an oblique magnetic field and Hall current," ISRN Mechanical Engineering, vol. 2011, Article ID 597172, 8 pages, 2011.

[36] S. Kaothekar and R. K. Chhajlani, "Gravitational instability of radiative plasma with finite Larmor radius corrections," Journal of Physics: Conference Series, vol. 365, no. 1, Article ID 012053, 2012. 

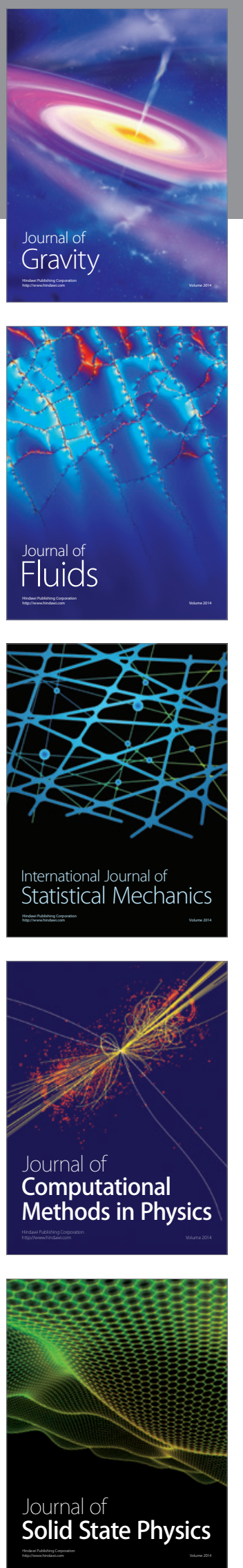

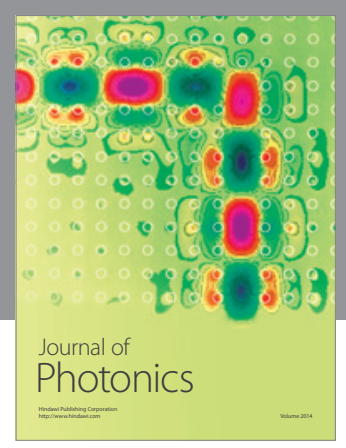

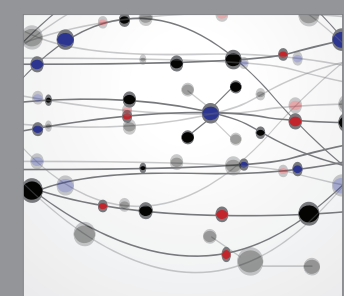

The Scientific World Journal
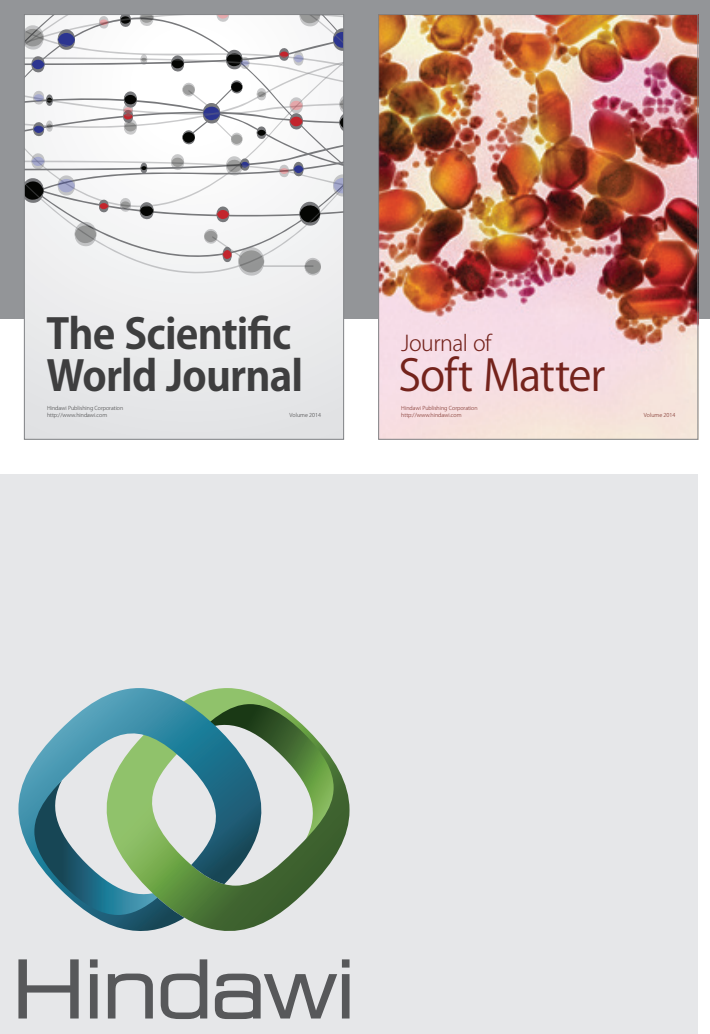

Submit your manuscripts at

http://www.hindawi.com
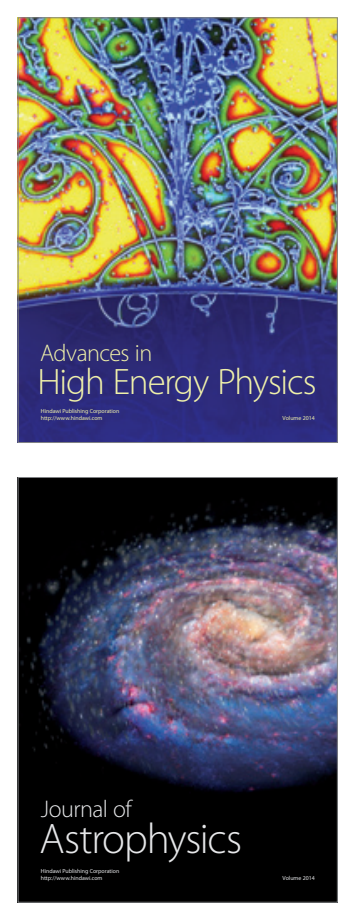
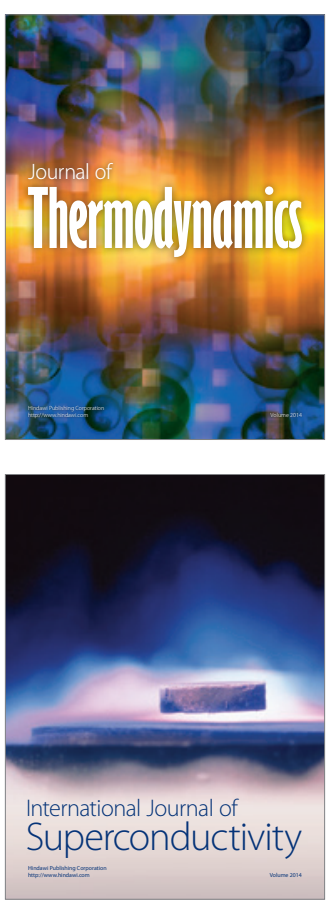
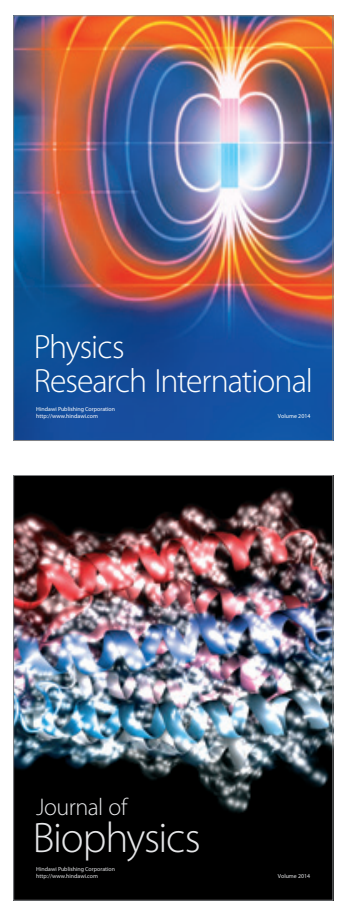
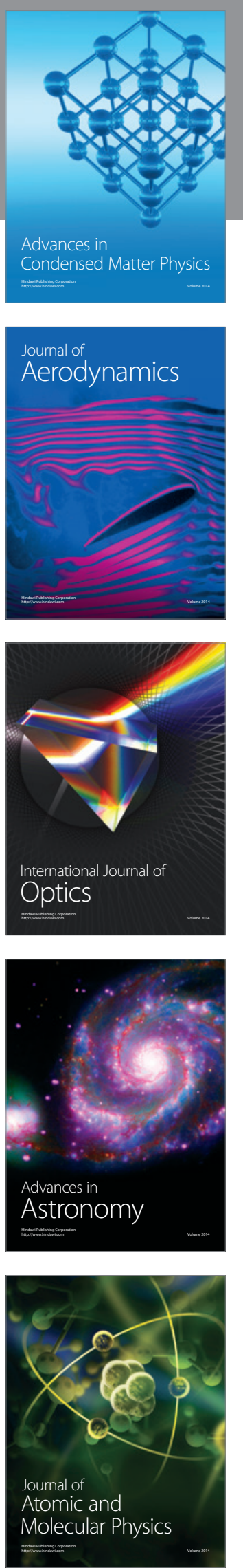